\title{
Kras in Organoids
}

\author{
Derek Cheng and David Tuveson \\ CSHL Cancer Center, Cold Spring Harbor Laboratory, Cold Spring Harbor, New York 11743 \\ Correspondence: dtuveson@cshl.edu
}

Oncogenic Kras are genetic dependencies for the majority of pancreatic and colorectal adenocarcinomas; however, much remains to be understood regarding its tropism to these carcinomas. Recently developed organoid technology presents a more representative model culture system for pancreatic and colon epithelial tissues as well as better fostering the culture of nonimmortalized cells than two-dimensional culture. These advantages enable cancer researchers to directly compare tumor and normal tissue models to better study tumor initiation as well as therapeutic efficacy. Although in vivo models better model the complexity of multiple cell types, the organoid system allows for easier genetic manipulations and isolation of specific cell types. Furthermore, syngeneic orthotopically transplanted organoids recapitulate tumor histologically and gene expression of the tumors from which they were derived. Thus, organoids may extend the use of genetically engineered mouse models. These advantages of organoid cultures allow for many questions, including but not limited to studying the interaction between different cell types within a tumor and elucidating dependencies of Kras-driven tumors.

$T_{0}^{\text {he }}$ he role of oncogenic Kras is especially of interest in the context of pancreatic ductal adenocarcinoma (PDAC) and colorectal (CRC) adenocarcinoma, as it is respectively mutated in $\sim 90 \%$ and $50 \%$ of cases (Gao et al. 2013). As a well-established oncogenic driver and dependency for tumor maintenance, Kras is a prime target for tumor-specific therapy (Tuveson et al. 2004; Jancík et al. 2010; Collins et al. 2012); however, many questions and challenges remain regarding Kras biology and Kras targeting. For example, despite being similar to the other Ras family members, Nras and Hras, only Kras has a higher prevalence of being mutated in the tumors just mentioned. Perhaps there are protein interactors and effectors that are specific to Kras that have yet to be discovered and can account for these differences. It also remains to be understood why the prevalence of Kras mutations in these specific cancer types is higher than in other cancers. This raises the question of whether Kras must be studied in a physiologically relevant model system that recapitulates traits of epithelial tumors and their corresponding normal cells of origin.

\section{ORGANOID CULTURE}

Organoid culture, which highly supports the growth of epithelial cells and preserves their functions, has been recently used to study Kras-related diseases (Sato et al. 2011; Li et al. 2014; Boj et al. 2015). Organoid culture was first

Editors: Linda VanAelst, Julian Downward, and Frank McCormick

Additional Perspectives on Ras and Cancer in the 21st Century available at www.perspectivesinmedicine.org

Copyright (C) 2018 Cold Spring Harbor Laboratory Press; all rights reserved; doi: 10.1101/cshperspect.a031575

Cite this article as Cold Spring Harb Perspect Med 2018;8:a031575 
developed for intestinal epithelial cell growth with $\operatorname{Lgr}^{+}$intestinal stem cells, and has now been adapted for other tissue applications (Dutta et al. 2017). Because of the various culture conditions and methods established from different laboratories, a standard definition of "organoid" has not been set. However, to be called an organoid, the culture must show organ-like properties such as being able to selforganize into a functional structure mimicking the architecture of the organ from which they were derived (Sato et al. 2009; Boj et al. 2015; Baker et al. 2016). To achieve a defined architecture, organoid cells are embedded in or grown on top of semisolid matrices such as agarose, collagen, or Matrigel. It has been suggested that organoids be reserved for three-dimensional (3D) cultures that are generated directly from dissociated primary tissue, embryonic stem, or iPS cells differentiated in vitro and propagated in a matrix rather than cells initially cultured as monolayers and assayed in 3D culture (Petersen et al. 1992). Organoid culture conditions have been established from both mouse and human gastrointestinal tissues, including intestinal (Sato et al. 2009), gastric (Barker et al. 2010), colon (Sato et al. 2011), pancreatic (Huch et al. 2013a; Boj et al. 2015), hepatic (Huch et al. 2013b), prostate (Gao et al. 2014), and breast (Rowald et al. 2016).

Organoid culture has some advantages over monolayer in vitro culture methods as well as in vivo models. In contrast to two-dimensional (2D) culture, cells in organoid culture maintain tissue structure by self-organizing and retaining features of polarity, with the basal side in contact with the Matrigel and an apical side toward the lumen of the organoid sphere (Sato et al. 2009; Huch et al. 2013b; Boj et al. 2015). Additionally, the organoid culture can be established with higher efficiency than 2D cell lines (Boj et al. 2015). More importantly, they allow for growth of nontransformed normal cells, whereas the 2D culture of these cells remains difficult. Organoid cultures can serve as a complementary tool, as well as an extension to genetically engineered mouse models. As an in vitro model system, organoids can be easily propagated and genetically manipulated to study specific targets com- pared with in vivo models. Syngeneic orthotopically transplanted organoids show architecture that resembles the tissue from which they were derived and therefore offer an expedited in vivo model system (Boj et al. 2015). These advantages make organoid culture a flexible tool to answer important questions, including how oncogenic Kras affects pancreatic ductal and colon epithelial cells.

\section{Kras IN MURINE PANCREATIC ORGANOIDS}

Since Kras mutations occur in $>90 \%$ of PDACs, the most common type of pancreatic cancer, these mutations are believed to be the driver of most PDAC cases (Almoguera et al. 1988). Therefore, the study of oncogenic Kras in pancreatic cells is ideal and, fortunately, organoids can increase the use of existing genetically engineered Kras-driven PDAC mouse models. Historically, a major challenge of studying Krasdriven cancer has been the difficulty in comparing in vitro cells with and without activated Kras in a disease-relevant context. Normal pancreatic ductal cells require immortalization for growth in 2D and would need wild-type (WT) and mutant Kras exogenously expressed (Furukawa et al. 1996). To address this hurdle, LSL-Kras mouse embryonic fibroblasts (MEFs) may be used to study the effect of the presence of oncogenic Kras. However, experiments should ideally be performed in a more relevant cell type such as pancreatic ductal cells. To model PDAC progression, $\mathrm{Kras}^{+/ \mathrm{LSL}-\mathrm{G} 12 \mathrm{D}}$; Pdx1-Cre (KC) mice were designed to express mutant Kras ${ }^{\mathrm{G} 12 \mathrm{D}}$ endogenously in the pancreas. These mice develop preinvasive ductal lesions that resemble human PanINs and, upon aging, can stochastically develop primary and metastatic PDAC (Hingorani et al. 2003). Although KC mice serve as a reliable source for murine PanIN tissue, $\mathrm{Kras}^{+} / \mathrm{LSL}^{\mathrm{G} 12 \mathrm{D}}$; $\operatorname{Trp} 53^{+} / \mathrm{LSL}-$ R172H; Pdx1-Cre (KPC) mice further expedite PDAC formation, providing a source for murine PDAC tumors and metastases (Hingorani et al. 2005). Together, WT, KC, and KPC mice cover the spectrum of PDAC progression from normal to PanIN, PDAC, and metastasis. Although some comparisons can be made between the 
tissues of normal, $\mathrm{KC}$, and $\mathrm{KPC}$ mice, there is still a demand for in vitro systems to more efficiently study the effects of genetic manipulation and drug response in the form of screens, and organoids have begun to meet these needs.

\section{Kras-Driven Mouse PanIN and Tumor Organoids Compared with Normal}

Boj et al. (2015) have shown that normal mouse and human pancreatic ductal cells as well as PanIN and tumor counterparts can be maintained in organoid culture for $>30$ passages. The ability to culture normal ductal cells enables the comparison of Kras WT cells with Kras mutant cells derived from tumor and PanIN lesions. It also enables the generation of isogenic Kras lines for delineating the biological consequences of acute Kras activation.

Organoids generated from WT, KC, and $\mathrm{KPC}$ mice serve as in vitro tools that complement their respective in vivo models. Genetic manipulation, large-scale screens, and characterization is often more feasible in in vitro systems because of the ability to propagate cells in a well-controlled setting. Organoids generated from normal ducts from WT mice, PanIN from $\mathrm{KC}$ mice, and tumor from KPC mice designated as $\mathrm{mN}, \mathrm{mP}$, and $\mathrm{mT}$, respectively, were established for this purpose. These organoids were verified to express genes of the ductal lineage such as CK19 and Sox9 (Cleveland et al. 2012). mP organoids also up-regulated genes that are associated with a PanIN disease state, such as Muc5ac, Muc6, and Tff1. As expected from expressing Kras ${ }^{\mathrm{G} 12 \mathrm{D}}$, mT organoids had increased levels of Kras-GTP but, interestingly, there was also elevated expression of the Kras protein (Boj et al. 2015). Although there is evidence from genetically engineered mouse models that mitogen-activated protein kinase (MAPK)/ERK and PI3K/AKT signaling may drive PDAC progression (Collisson et al. 2012; Eser et al. 2013), mT and metastatic (mM) organoids revealed Erk and Akt phosphorylation similar to that of normal organoids but with relatively increased downstream S6 phosphorylation. The lack of phosphorylation of ERK in organoids is also consistent with what is seen in
PanIN lesions of KC mice and LSL-Kras MEFs and may be caused by negative feedback mechanisms (Karreth and Tuveson 2009).

Culture conditions may not only affect cell signaling but also more dramatic changes such as karyotypic stability. Loss of heterozygosity (LOH) for Trp53 has been commonly seen in mouse PDAC based on studies of 2D cell lines (Hingorani et al. 2005). Interestingly, murine pancreatic tumor cells cultured as organoids maintain a stable diploid karyotype but the same cells plated as 2D cell lines spontaneously become aneuploid and lose their WT copy of p53 (Boj et al. 2015). This LOH after plating mouse tumor cells in $2 \mathrm{D}$ suggests that culture conditions can greatly affect the genomic makeup of the cells, as well as the expression of genes. 2D culture may select for p53 LOH cells and, therefore, may not accurately reflect tumor heterogeneity. Because the number of copies of WT and mutant Kras has been shown to affect MAPK dependence, organoid culture may provide a way to study tumor cells without chromosomal imbalance (Burgess et al. 2017).

Organoids are also useful for transplantation experiments. Mouse organoids can be orthotopically transplanted into syngeneic C57Bl/ 6 or $\mathrm{Nu} / \mathrm{Nu}$ mice. Although transplanted $\mathrm{mN}$ organoids form ductal structures resembling normal ductal architecture comprised of simple cuboidal cells, mP organoids formed small cysts lined with simple cuboidal cells as well as patches of mucin-containing columnar epithelial cells resembling PanIN morphology. Like PanIN tissue, these transplanted dysplastic cells had increased proliferation and stromal response. When mT organoids are orthotopically transplanted, they initially form early and late PanIN-like lesions and eventually develop into invasive or metastatic PDAC. Although mT organoids take several months to progress to invasive PDAC, organoids derived from $\mathrm{mM}$ quickly engraft as invasive PDAC. In addition to displaying dysplasia, transplanted tumor organoids also induce a stroma response leading to lowly vascularized tumors. The organoid transplantation model retains many of the characteristics of autochthonous PanIN and PDAC tissue and offers an alternative PDAC progression model. 
D. Cheng and D. Tuveson

\section{Kras Activation in Mouse Pancreatic Organoids}

The ability to culture normal organoids permits the generation of isogenic cultures allowing for the study of Kras activation. To generate these lines, organoids derived from pancreatic ducts harboring the conditional Kras ${ }^{\text {LSL-G12D }}$ allele were infected with adenoviral-Cre recombinase and were compared with the control-infected organoids (Boj et al. 2015). Kras ${ }^{\text {G12D }}$-expressing organoids maintained expression of genes specific to ductal cells rather than acinar and endocrine lineages but showed an increase in Kras expression compared with control-infected organoids. Kras ${ }^{\mathrm{G} 12 \mathrm{D}}$-expressing organoids also showed a greater proliferation rate compared with control-infected organoids. Additionally, transplanting $\mathrm{Kras}^{\mathrm{G} 12 \mathrm{D}}$-expressing organoids gave rise to PanIN-like lesions, whereas controlinfected organoids formed normal-appearing ductal architecture. These PanIN-like lesions showed tall columnar morphology and produced mucin. These findings are consistent with the observation that endogenous Kras ${ }^{\mathrm{G} 12 \mathrm{D}}$ expression is sufficient in giving rise to PanIN lesions in mice (Hingorani et al. 2003). Because the organoid cultures described above support ductal cells and no other lineages, these studies show that ductal cells, when expressing Kras ${ }^{\text {G12D }}$, are competent in forming PanIN lesions. Similar to $\mathrm{mP}$ organoids, which also express Kras ${ }^{\mathrm{G} 12 \mathrm{D}}$, these $\mathrm{Kras}^{\mathrm{G} 12 \mathrm{D}}$ organoids also up-regulate genes associated with early PanIN lesions (Muc5ac, Muc6, Tff1) when compared with control-infected organoids. RNA sequencing characterization of $\mathrm{mN}, \mathrm{mP}$, and $\mathrm{mT}$ revealed that several genes were up-regulated in $\mathrm{mP}$ and $\mathrm{mT}$ organoids. Of these, Gcnt1, Gcnt3, Acsm3, Agr2, Syt16, Nt5e, and Ugdh, were also up-regulated in Kras ${ }^{\mathrm{G} 2 \mathrm{D}}$ organoids compared to infected control organoids, suggesting that up-regulation of these genes is downstream from Kras activation. In short, activation of Kras in vitro can yield PanIN-like organoids, so it follows that PanIN-associated cellular changes may be Kras-driven behaviors. Gene set enrichment analysis (GSEA) on the RNA-seq and proteomic data of mouse organoids revealed that $\mathrm{mP}$ organoids up-regulated genes are involved in glutathione metabolism, which is involved with redox regulation (Boj et al. 2015).

The study of Kras ${ }^{\text {G12D }}$ using organoids has particularly uncovered the dependencies of Kras cells on the oxidative state of pancreatic tumor cells. Kras activation has been shown to induce Nrf2 protein expression and/or its target gene, Nqo1, in KPC mouse tumors and their primary cultures (DeNicola et al. 2011). With regard to its relevance in human disease, Nrf2 protein is increased in human tumor organoids compared with normal counterparts (Chio et al. 2016). As a master regulator of an antioxidant response, the transcription factor Nrf2 keeps reactive oxygen species (ROS) levels down and is a dependency of Kras-driven PanIN formation (DeNicola et al. 2011). To investigate the biochemical effects and function of Nrf2 in Kras ${ }^{\mathrm{G} 12 \mathrm{D}}$-driven tumor maintenance, Chio et al. (2016) performed Nrf2 knockdown and ablation experiments in both human and mouse organoid models. As seen in the KC and KPC mouse organoid models, Kras ${ }^{\mathrm{G} 12 \mathrm{D}}$ induces the Nrf2 antioxidant program, leading to an increase in intracellular glutathione (GSH) and decreased levels of ROS; features reverted on NRF2 ablation (Chio et al. 2016). Nrf2 knockdown in human organoids inhibits the growth of some human tumor organoids (KRAS mutant) although being tolerated by human normal organoids (KRAS WT) (Chio et al. 2016). Interestingly, the surviving tumor organoids showed elevated ROS and a decrease in cell proliferation, which was alleviated by the addition of antioxidant NAC. These Nrf2-depletion experiments suggest that Kras mutant tumor organoids depend more on Nrf2 for cell proliferation than Kras WT normal organoids, likely the result of elevation of ROS in tumor cells. Dependencies of Kras cells such as Nrf2 suppression of ROS as seen in organoids may elucidate novel therapeutic approaches in pancreatic cancer.

\section{Coculture Studies with Mouse Kras Organoids}

Organoids derived from KPC tumors are also a relevant model for studying cell-to-cell interactions. Organoid cultures allow us to identify dis- 
tinct populations of cancer-associated fibroblasts (CAFs) and confirm their presence in vivo. Although Kras is mutated in the epithelial cells that drive PDAC, up to $90 \%$ of the total volume of the tumor is made of desmoplasia, consisting of extracellular matrix, immune cells, vasculature, and cancer-associated fibroblasts (Moir et al. 2015). The organoid system allows for the study of interactions between carcinoma cells and pancreatic stellate cells (PSCs), which support such desmoplasia. Because the organoid system maintains spatial organization of cells, the phenomenon of heterogeneity in fibroblasts was discovered (Öhlund et al. 2017). PSCs and tumor organoids cocultured in Matrigel can proliferate in the presence of reduced media. Interestingly, the PSCs and tumor organoids promote the proliferation of each other. Although PSCs are relatively quiescent when plated alone in Matrigel, the PSCs show morphological changes and deposit collagen I when in close contact with tumor organoids. This PSC activation in vitro recapitulates the desmoplasia characteristic of PDAC (Öhlund et al. 2017). Although tumor organoids are capable of triggering PSC activation, it remains to be tested whether Kras mutant cells induce specific changes in PSCs that Kras WT cells do not.

In addition to PSCs, peritoneal mesothelial cells and adipocytes have been shown to affect the behavior of pancreatic tumor cells. In another coculture system, Abe et al. (2017) showed that $3 \mathrm{D}$ coculture and cotransplantation of peritoneal mesothelial cells with pancreatic cancer cells derived from KPC tumors lead to increased invasion of the cancer cells. Another cell-cell interaction relevant to cancer may be that of the cancer cell to neighboring adipocytes. Okumura et al. (2017) observed an increase in metastasis in KPC mice fed on a high-fat diet. This adipose tissue-derived conditioned media increased the migration, invasiveness, and gemcitabine resistance of KPC tumor cells. These changes were attributed to direct uptake of released fatty acids from adipocytes as seen with $3 \mathrm{D}$ culture experiments with conditioned media from adipose tissue embedded in collagen I (Okumura et al. 2017). It remains to be shown whether the interaction of KPC tumor organ- oids with PSC, peritoneal mesothelial cells, and adipocyctes are specific to Kras mutant cells, to tumor cells, or to proliferating epithelial cells in general.

\section{LESSONS FROM HUMAN PANCREATIC ORGANOIDS}

Human disease may be more complex than our currently used mouse models. Thus, human organoids are of interest to model PDAC as well as other cancers. Boj et al. (2015) have established human pancreatic organoid cultures from normal tissue $(\mathrm{hN})$, PDAC biopsies (hT), and fine needle aspirations (hFNA) and metastases (hM) with $>75 \%$ efficiency. Unsurprisingly, Kras was mutated in seven of eight organoids derived from tumor and both organoids derived from metastases; however, human PDAC is a complex disease. There are some notable differences between human organoids and mouse organoids. $\mathrm{hN}$ organoids can only be propagated for $\sim 20$ passages, causing some limitation to experiments that can be performed on them. hT organoids often grow as dysplastic tall columnar cells and the organoids can proliferate as filled spheres rather than the simple cuboidal lined cysts of $\mathrm{hN}$. Because organoids are grown in vitro, the culturing conditions can be controlled leading to identification of specific dependencies for growth factors and nutrients. In the case of human pancreatic ductal organoids, tumor organoids can be grown in the absence of epithelial growth factor (EGF), whereas normal organoids cannot (Boj et al. 2015). Likely, the expression of mutant Kras allows these organoids to become EGF-independent, whereas normal organoids remain EGF-dependent; however, this concept has yet to be validated in isogenic lines.

Although representative of the tumor from which they are derived, human tumor organoids vary in other mutations such as p53, SMAD4, and CDKN2A (Boj et al. 2015). Because of the heterogeneity of human tumors, isogenic experiments in a human context would be beneficial. Introducing oncogenic Kras ectopically via lentiviral infection and knocking out p53, CDKN2, and SMAD4 simultaneously by CRISPR in 
D. Cheng and D. Tuveson

$\mathrm{CD}_{133^{+}}$human pancreatic ductal cells and orthotopically transplanting these cells into the splenic lobe of pancreas of immunocompromised NOD SCID IL2rg null (NSG) mice was sufficient in PanIN-1 formation. The further introduction of ERBB2 overexpression into these organoids followed by transplantation causes formation of PanIN-2-like formation. Although this model system only recreated PanIN lesions, it is a transplantation model that uses human cells. Although human cells seem to require more mutations to drive transformation compared with the Kras and p53 in mouse organoids, it is consistent with how human organoids have also been shown to require more components in organoid media for propagation. These observations may suggest that the human disease may be more complex than the mouse model, and more genetic changes are required to supplement the driving Kras mutation in human disease.

\section{Kras IN COLON ORGANOIDS}

Kras is also a known CRC driver gene. Li et al. (2014) used the single air-liquid interface method to model malignancies of the pancreas, stomach, and colon. This organoid method cultures primary mouse epithelial and mesenchymal cells, yielding detailed in situ histologic end points for oncogenic transformation in vitro, and tumorigenicity after transplantation in vivo. Consistent with the findings from the KPC organoid system by Boj et al. (2015), K, P, and $\mathrm{KP}$ organoids also showed increased tumorigenicity when Cre expression activated Kras ${ }^{\text {G12D }}$ expression in various primary gastrointestinal epithelial cells (Li et al. 2014).

Because organoids recapitulate many Rasdriven phenomenon, organoids may provide a more informative model for screening Ras pathway inhibitors. Because nontransformed cells with WT Kras can be cultured alongside Kras mutant tumor cells, the organoid platform can better aid us in the search for targeted therapy with an effective therapeutic index. Verissimo et al. (2016) tested clinically used epidermal growth factor receptor (EGFR)-RAS-ERK pathway inhibitors in organoids derived from nor- mal colon tissue and colon cancer. They found features that are seen in in vivo data and clinical trials. Clinically, treatment of CRC tumors is dependent on Kras mutation status, namely, the effectiveness of EGFR inhibition against WT Kras tumors but not mutant Kras tumors. Interestingly, the drug effects were similar when comparing normal organoids to patient-derived CRC organoids with WT Kras. Drug resistance against all tested EGFR inhibitors correlated with the mutational status of KRAS, which is consistent with the EGF withdrawal experiments done in human pancreatic organoids mentioned earlier (Boj et al. 2015). MEK (selumetinib) and ERK (SCH772984) inhibitors were effective in all organoid lines tested. The combination of EGFR and MEK inhibition is additive and leads to more consistent inhibitory effects on the CRC organoids. Combination therapies such as dual pan-HER and MEK inhibition as well as dual MEK and ERK inhibition, are in clinical trials. Although the CRC organoid model show synergistic effects of combination therapy suppressing the growth of Ras mutant organoids, this leads to cell-cycle arrest rather than cell death but sensitizes cells to $\mathrm{Bcl} 2$. Although the tested drugs did not selectively inhibit tumor organoids over normal organoids, this study shows that the organoid platform is a preclinical drug-screening tool that is representative of patient tumor response. As new antiRas therapies such as direct allosteric inhibitors and drugs that block Kras from interacting with other proteins are developed, organoids may be the ideal model system in which to test their efficacy preclinically (Maurer et al. 2012; Ostrem et al. 2013; Shima et al. 2013).

\section{ACHIEVING A BETTER MODEL}

Ras continues to be a highly important topic because Kras is a driver of many tumors and remains without effective therapies that target it directly or indirectly in the clinic. Genetically engineered LSL-Kras ${ }^{\text {G12D }}$ mouse models have enabled the study of endogenously expressed oncogenic Kras, and now organoid models have extended the use of this model and enabled the study of Kras ${ }^{\mathrm{G} 12 \mathrm{D}}$ in epithelial cells. Organ- 
oids provide an in vitro cancer progression model from normal cells to PanIN, tumor, and metastatic cells. Progression models are essential to studying cancer as the time to diagnosis of cancer often limits what physicians can do for their patients. Being able to dissect the biology of early tumors and high-risk PanIN lesions, researchers may be able to identify early biomarkers leading to improved diagnostics or targets for cancer prevention, both of which could lead to better patient outcomes. Furthermore, organoids provide a way to study the interaction of cancer cells with neighboring cells such as cancer-associated fibroblasts. As the model becomes even better characterized, it will be interesting to see other coculture systems to better model the tumors, which are complex in the cell types present. With the ability of propagating Kras WT and Kras ${ }^{\mathrm{G} 12 \mathrm{D}}$ organoids under the same conditions, screening for mutant Kras-specific therapies can be facilitated and the confounding factors inherent when comparing across cell lines are minimized. The LSL-Kras ${ }^{\text {G12D }}$ model still has the caveat of expressing one allele of WT Kras when Cre is not present and one allele each of WT and mutant when Cre is present. Therefore, the next advance in building a better Kras model to study pancreatic disease may be a system where comparisons between Kras WT-expressing cells and Kras ${ }^{\mathrm{G} 12 \mathrm{D}}$-expressing cells will have both alleles functional. Theoretically, this could be accomplished with the advances in CRISPR technology in the organoid model system. In summary, organoids open the door to ask many Kras-related questions that, when answered, could impact how we treat Kras-driven cancers.

\section{REFERENCES}

Abe T, Ohuchida K, Koikawa K, Endo S, Okumura T, Sada M, Horioka K, Zheng B, Moriyama T, Nakata K, et al. 2017. Cancer-associated peritoneal mesothelial cells lead the formation of pancreatic cancer peritoneal dissemination. Int J Oncol 50: 457-467.

Almoguera C, Shibata D, Forrester K, Martin J, Arnheim N, Perucho M. 1988. Most human carcinomas of the exocrine pancreas contain mutant c-K-ras genes. Cell 53: 549-554.
Baker LA, Tiriac H, Clevers H, Tuveson DA. 2016. Modeling pancreatic cancer with organoids. Trends Cancer 2: 176190.

Barker N, Huch M, Kujala P, van de Wetering M, Snippert HJ, van Es JH, Sato T, Stange DE, Begthel H, van den Born M, et al. 2010. Lgr5 ${ }^{+v e}$ stem cells drive self-renewal in the stomach and build long-lived gastric units in vitro. Cell Stem Cell 6: 25-36.

Boj SF, Hwang CI, Baker LA, Chio II, Engle DD, Corbo V, Jager M, Ponz-Sarvise M, Tiriac H, Spector MS, et al. 2015. Organoid models of human and mouse ductal pancreatic cancer. Cell 160: 324-338.

Burgess MR, Hwang E, Mroue R, Bielski CM, Wandler AM, Huang BJ, Firestone AJ, Young A, Lacap JA, Crocker L, et al. 2017. KRAS allelic imbalance enhances fitness and modulates MAP kinase dependence in cancer. Cell 168: 817-829.e15.

Chio IIC, Jafarnejad SM, Ponz-Sarvise M, Park Y, Rivera K, Palm W, Wilson J, Sangar V, Hao Y, Öhlund D, et al. 2016 NRF2 promotes tumor maintenance by modulating mRNA translation in pancreatic cancer. Cell 166: 963976.

Cleveland MH, Sawyer JM, Afelik S, Jensen J, Leach SD. 2012. Exocrine ontogenies: On the development of pancreatic acinar, ductal and centroacinar cells. Semin Cell Dev Biol 23: 711-719.

Collins MA, Brisset JC, Zhang Y, Bednar F, Pierre J, Heist KA, Galbán CJ, Galbán S, di Magliano MP. 2012. Metastatic pancreatic cancer is dependent on oncogenic Kras in mice. PLoS ONE 7: e49707.

Collisson EA, Trejo CL, Silva JM, Gu S, Korkola JE, Heiser LM, Charles RP, Rabinovich BA, Hann B, Dankort D, et al. 2012. A central role for $\mathrm{RAF} \rightarrow \mathrm{MEK} \rightarrow \mathrm{ERK}$ signaling in the genesis of pancreatic ductal adenocarcinoma. Cancer Discov 2: 685-693.

DeNicola GM, Karreth FA, Humpton TJ, Gopinathan A, Wei C, Frese K, Mangal D, Yu KH, Yeo CJ, Calhoun ES, et al. 2011. Oncogene-induced Nrf2 transcription promotes ROS detoxification and tumorigenesis. Nature 475: 106-109109.

Dutta D, Heo I, Clevers H. 2017. Disease modeling in stem cell-derived 3D organoid systems. Trends Mol Med 23: 393-410.

Eser S, Reiff N, Messer M, Seidler B, Gottschalk K, Dobler M, Hieber M, Arbeiter A, Klein S, Kong B, et al. 2013. Selective requirement of PI3K/PDK1 signaling for Kras oncogene-driven pancreatic cell plasticity and cancer. Cancer Cell 23: 406-420.

Furukawa T, Duguid WP, Rosenberg L, Viallet J, Galloway DA, Tsao MS. 1996. Long-term culture and immortalization of epithelial cells from normal adult human pancreatic ducts transfected by the E6E7 gene of human papilloma virus 16. Am J Pathol 148: 1763-1770.

Gao J, Aksoy BA, Dogrusoz U, Dresdner G, Gross B, Sumer SO, Sun Y, Jacobsen A, Sinha R, Larsson E, et al. 2013. Integrative analysis of complex cancer genomics and clinical profiles using the cBioPortal. Sci Signal 6: 1.

Gao D, Vela I, Sboner A, Iaquinta PJ, Karthaus WR, Gopalan A, Dowling C, Wanjala JN, Undvall EA, Arora VK, et al. 2014. Organoid cultures derived from patients with advanced prostate cancer. Cell 159: 176-187. 
D. Cheng and D. Tuveson

Hingorani SR, Petricoin EF, Maitra A, Rajapakse V, King C, Jacobetz MA, Ross S, Conrads TP, Veenstra TD, Hitt BA, et al. 2003. Preinvasive and invasive ductal pancreatic cancer and its early detection in the mouse. Cancer Cell 4: 437-450.

Hingorani SR, Wang L, Multani AS, Combs C, Deramaudt TB, Hruban RH, Rustgi AK, Chang S, Tuveson DA. 2005. $\operatorname{Trp} 53^{R 172 H}$ and Kras ${ }^{G 12 D}$ cooperate to promote chromosomal instability and widely metastatic pancreatic ductal adenocarcinoma in mice. Cancer Cell 7: 469-483.

Huch M, Bonfanti P, Boj SF, Sato T, Loomans CJ, van de Wetering M, Sojoodi M, Li VS, Schuijers J, Gracanin A, et al. 2013a. Unlimited in vitro expansion of adult bipotent pancreas progenitors through the Lgr5/R-spondin axis. EMBO J 32: 2708-2721.

Huch M, Dorrell C, Boj SF, van Es JH, Li VS, van de Wetering M, Sato T, Hamer K, Sasaki N, Finegold MJ, et al. 2013b. In vitro expansion of single $\mathrm{Lgr}^{+}$liver stem cells induced by Wnt-driven regeneration. Nature 494: 247-250.

Jancík S, Drábek J, Radzioch D, Hajdúch M. 2010. Clinical relevance of KRAS in human cancers. J Biomed Biotechnol doi: 10.1155/2010/150960.

Karreth FA, Tuveson DA. 2009. Modelling oncogenic Ras/Raf signalling in the mouse. Curr Opin Genet Dev 19: 4-11.

Li XN, Nadauld L, Ootani A, Corney DC, Pai RK, Gevaert O, Cantrell MA, Rack PG, Neal JT, Chan CW, et al. 2014. Oncogenic transformation of diverse gastrointestinal tissues in primary organoid culture. Nat Med 20: 769-777.

Maurer T, Garrenton LS, Oh A, Pitts K, Anderson DJ, Skelton NJ, Fauber BP, Pan B, Malek S, Stokoe D, et al. 2012. Small-molecule ligands bind to a distinct pocket in Ras and inhibit SOS-mediated nucleotide exchange activity. Proc Natl Acad Sci 109: 5299-5304.

Moir JA, Mann J, White SA. 2015. The role of pancreatic stellate cells in pancreatic cancer. Surg Oncol 24: 232-238.

Öhlund D, Handly-Santana A, Biffi G, Elyada E, Almeida AS, Ponz-Sarvise M, Corbo V, Oni TE, Hearn SA, Lee EJ, et al. 2017. Distinct populations of inflammatory fibroblasts and myofibroblasts in pancreatic cancer. J Exp Med 214: 579-596.

Okumura T, Ohuchida K, Sada M, Abe T, Endo S, Koikawa K, Iwamoto C, Miura D, Mizuuchi Y, Moriyama T, et al.
2017. Extra-pancreatic invasion induces lipolytic and fibrotic changes in the adipose microenvironment, with released fatty acids enhancing the invasiveness of pancreatic cancer cells. Oncotarget 8: 18280-18295.

Ostrem JM, Peters U, Sos ML, Wells JA, Shokat KM. 2013. K-Ras ${ }^{\text {G12C }}$ inhibitors allosterically control GTP affinity and effector interactions. Nature 503: 548-551.

Petersen OW, Rønnov-Jessen L, Howlett AR, Bissell MJ. 1992. Interaction with basement membrane serves to rapidly distinguish growth and differentiation pattern of normal and malignant human breast spithelial cells. Proc Natl Acad Sci 89: 9064-9068.

Rowald K, Mantovan M, Passos J, Buccitelli C, Mardin BR, Korbel JO, Jechlinger M, Sotillo R. 2016. Negative selection and chromosome instability induced by Mad2 overexpression delay breast cancer but facilitate oncogene-independent outgrowth. Cell Rep 15: 2679-2691.

Sato T, Vries RG, Snippert HJ, van de Wetering M, Barker N, Stange DE, van Es JH, Abo A, Kujala P, Peters PJ, et al. 2009. Single Lgr5 stem cells build crypt-villus structures in vitro without a mesenchymal niche. Nature 459: 262265.

Sato T, Stange DE, Ferrante M, Vries RG, Van Es JH, Van den Brink S, Van Houdt WJ, Pronk A, Van Gorp J, Siersema PD, et al. 2011. Long-term expansion of epithelial organoids from human colon, adenoma, adenocarcinoma, and Barrett's epithelium. Gastroenterology 141: 1762-1772.

Shima F, Yoshikawa Y, Ye M, Araki M, Matsumoto S, Liao J, Hu L, Sugimoto T, Ijiri Y, Takeda A, et al. 2013. In silico discovery of small-molecule Ras inhibitors that display antitumor activity by blocking the Ras-effector interaction. Proc Natl Acad Sci 110: 8182-8187.

Tuveson DA, Shaw AT, Willis NA, Silver DP, Jackson EL, Chang S, Mercer KL, Grochow R, Hock H, Crowley D, et al. 2004. Endogenous oncogenic K-ras ${ }^{G 12 D}$ stimulates proliferation and widespread neoplastic and developmental defects. Cancer Cell 5: 375-387.

Verissimo CS, Overmeer RM, Ponsioen B, Drost J, Mertens S, Verlaan-Klink I, Gerwen BV, van der Ven M, Wetering MV, Egan DA, et al. 2016. Targeting mutant RAS in patient-derived colorectal cancer organoids by combinatorial drug screening. eLife 5: e18489. 


\section{$\&_{\mathrm{CSH}}^{\infty} \&$ Cold Spring Harbor

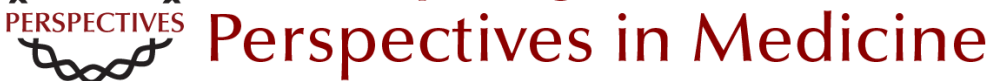

\section{Kras in Organoids}

Derek Cheng and David Tuveson

Cold Spring Harb Perspect Med 2018; doi: 10.1101/cshperspect.a031575 originally published online January 8, 2018

\section{Subject Collection Ras and Cancer in the 21st Century}

Targeting Ras with Macromolecules Dehua Pei, Kuangyu Chen and Hui Liao

Ras-Specific GTPase-Activating Proteins-Structures, Mechanisms, and Interactions Klaus Scheffzek and Giridhar Shivalingaiah

Ras-Mediated Activation of the Raf Family Kinases Elizabeth M. Terrell and Deborah K. Morrison

Posttranslational Modifications of RAS Proteins Ian Ahearn, Mo Zhou and Mark R. Philips

Kras in Organoids Derek Cheng and David Tuveson

KRAS: The Critical Driver and Therapeutic Target for Pancreatic Cancer Andrew M. Waters and Channing J. Der

The K-Ras, N-Ras, and H-Ras Isoforms: Unique Conformational Preferences and Implications for Targeting Oncogenic Mutants Jillian A. Parker and Carla Mattos

PI3K: A Crucial Piece in the RAS Signaling Puzzle Agata Adelajda Krygowska and Esther Castellano
MRAS: A Close but Understudied Member of the RAS Family Lucy C. Young and Pablo Rodriguez-Viciana

The Interdependent Activation of

Son-of-Sevenless and Ras Pradeep Bandaru, Yasushi Kondo and John Kuriyan

Targeting the MAPK Pathway in RAS Mutant Cancers

Sarah G. Hymowitz and Shiva Malek

Ras and the Plasma Membrane: A Complicated

Relationship

Yong Zhou, Priyanka Prakash, Alemayehu A. Gorfe, et al.

Kras and Tumor Immunity: Friend or Foe? Jane Cullis, Shipra Das and Dafna Bar-Sagi

Synthetic Lethal Vulnerabilities in KRAS-Mutant Cancers Andrew J. Aguirre and William C. Hahn

Efforts to Develop KRAS Inhibitors Matthew Holderfield

Genetically Engineered Mouse Models of K-Ras-Driven Lung and Pancreatic Tumors: Validation of Therapeutic Targets Matthias Drosten, Carmen Guerra and Mariano Barbacid

For additional articles in this collection, see http://perspectivesinmedicine.cshlp.org/cgi/collection/ 